\title{
Sub-analysis of geographical variations in the 2-year observational COPTIMIZE trial of patients with relapsing-remitting multiple sclerosis converting to glatiramer acetate
}

\author{
Tjalf Ziemssen $^{1 *}$ and Yossi Gilgun-Sherki ${ }^{2}$
}

\begin{abstract}
Background: Studies suggest that patients with relapsing-remitting multiple sclerosis (RRMS) who fail to benefit from a disease-modifying treatment (DMT) may benefit from converting to another DMT class. COPTIMIZE was a 24-month observational study designed to assess the disease course of patients converting to glatiramer acetate (GA) 20 mg daily from another DMT and the association of disease characteristics and reasons for converting. This sub-analysis was to determine if any findings varied by three geographic locations: Latin America (LA), Canada and Western Europe (CWE), and Eastern Europe (EE).
\end{abstract}

Methods: A total of 668 patients were included (263 LA, 248 CWE, 157 EE) in an analysis of annualized relapse rate (ARR) and annualized rate of deterioration (ARD), as well as secondary endpoints including reason for DMT switch and changes in disability and fatigue scores. Repeated-measures analysis of variance and log transformation were used to analyze ARR and ARD, whereas the Wilcoxon signed rank test was used for secondary endpoints.

Results: The sub-analysis of treatment outcomes stratified by region showed that Latin American patients had higher ARR before conversion to GA compared with patients from the other two areas and subsequently experienced the largest reduction in ARR. Latin American patients also had higher baseline rates of comorbidities and relapses with incomplete remissions and improved more than those in the other two regions based on measures of fatigue, quality of life, depression, and cognition scores. Latin American patients also generally had a better perception of the benefits associated with their conversion to GA in terms of efficacy and adverse events.

Conclusions: These findings indicate that, in RRMS patients, converting to GA is associated with positive treatment outcomes regardless of geographic location. However, the reasons for converting and the type and degree of any associated benefits appear to vary depending on various factors, including patients' geographical location.

Keywords: Multiple sclerosis, RRMS, Glatiramer acetate, Demographics

\section{Background}

Multiple sclerosis (MS) is a chronic relapsing disorder of the central nervous system characterized by inflammation, multifocal demyelination, and neuronal and axonal damage [1]. The majority of MS patients initially present with relapsing-remitting MS (RRMS) that frequently

\footnotetext{
* Correspondence: tjalf.ziemssen@uniklinikum-dresden.de

${ }^{1}$ Center of Clinical Neuroscience, Neurological University Clinic, University Clinic Carl Gustav Carus, University of Technology Dresden, Fetscherstraße 74, D-01307 Dresden, Germany

Full list of author information is available at the end of the article
}

develops to a progressive disease course [1]. The prevalence of MS varies according to geographic location from 10 to 20 per 100,000 in Central and South America to >30 per 100,000 in northern Europe and North America [2].

Immunomodulating disease-modifying therapies (DMTs) have been shown to improve multiple measures of disease activity in RRMS patients, including the annualized relapse rate (ARR), proportion of relapse-free individuals, and accumulation of T2 lesion burden [3-5]. However, these agents are only partially effective in controlling disease progression; studies have reported treatment interruption or

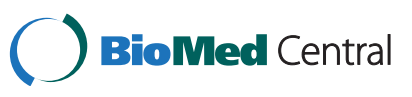

(c) 2015 Ziemssen and Gilgun-Sherki. Open Access This article is distributed under the terms of the Creative Commons Attribution 4.0 International License (http://creativecommons.org/licenses/by/4.0/), which permits unrestricted use, distribution, and reproduction in any medium, provided you give appropriate credit to the original author(s) and the source, provide a link to the Creative Commons license, and indicate if changes were made. The Creative Commons Public Domain Dedication waiver (http://creativecommons.org/publicdomain/zero/1.0/) applies to the data made available in this article, unless otherwise stated. 
discontinuation because of lack of tolerability, progression of disability, or inadequate clinical response [6]. Additionally, the development of neutralizing antibodies, specifically with interferon $\beta$ products (IFNs) and natalizumab [7], can interfere with the biologic response [8].

Converting to another DMT class represents one treatment strategy for MS patients with an inadequate response to first-line treatments or intolerant side effects [9]. Expert guidance on the specific steps of a conversion has been reported [10]; however, the lack of information on outcomes in different populations [11] results in limited guidance on regional patient considerations.

The COPTIMIZE study was designed to monitor clinical outcomes after converting from failing or ineffective DMT therapy for RRMS to glatiramer acetate (GA) in a prospective way. GA is approved in 57 countries as a $20 \mathrm{mg}$ daily subcutaneous (s.c.) injection for reducing relapse frequency in patients with RRMS. It has long-term efficacy and safety data, with the longest continuous treatment exposure of more than 20 years $[12,13]$. The primary results of COPTIMIZE, presented elsewhere [14], indicate that a conversion to GA is associated with positive treatment outcomes and that the benefits vary depending on patients' reasons for changing. The primary objective of the present sub-analysis is to determine whether benefits associated with converting to GA were affected by therapeutic strategies and patient selection in different geographic locations: Latin America (LA), Canada and Western Europe (CWE), and Eastern Europe (EE).

\section{Methods}

\section{Study design}

Study design, patient eligibility criteria, and conduct of the COPTIMIZE have been previously reported [14]. Briefly, this post-hoc subgroup analysis attempted to describe any variation in results that might exist between three predefined geographical areas: LA (Argentina, Brazil, Chile, Mexico, Venezuela), CWE (Belgium, Canada, Denmark, France, Greece, Ireland, Portugal, Netherlands, Norway, Sweden), and EE (Hungary, Romania, Slovakia). Countries were grouped into regions based on similarity of healthcare systems, physician approaches [15], available treatment options $[10,16]$, and epidemiological characteristics of the population (disease prevalence, demographics, etc.) [2]. All countries investigated in this observational study reported the use of IFN- $\beta$ and GA at baseline, with no anticipated systematic differences between regions. This study was conducted in accordance with the 18th World Medical Assembly (Helsinki) recommendations and amendments, as well as guidelines for Good Epidemiology Practice. The study protocol was approved by the institutional review boards and independent ethics committees at all participating study locations in each individual country; each site ensured all necessary regulatory submissions in accordance with local regulations including local data protection regulations. All patients provided informed, written consent according to local independent review board ruling.

\section{Study endpoints}

The primary objective was to assess the disease course in RRMS patients converting from IFN treatment to GA as measured by the primary endpoints of ARR and annualized rate of deterioration (ARD) (confirmed progression of Expanded Disability Status Scale [EDSS]/worsening mobility scores). Secondary data collected included reasons for DMT conversion, characteristics of patients failing to benefit from previous DMT, and change in EDSS and modified fatigue impact scale (MFIS) scores. Also recorded were quality of life (QoL) changes following GA conversion as measured by the Functional Assessment of Multiple Sclerosis (FAMS), cognition changes as evaluated by the Paced Auditory Serial Addition Test (PASAT), depression as measured by Centre for Epidemiologic Studies Depression (CES-D) scale, and change in adverse events (AEs) following the conversion.

\section{Statistical analyses}

Statistical analyses of parameters in this observational study required comparison of at least two endpoint measures, pre- and post-GA conversion, with data represented by descriptive procedures and figures, if necessary. Adjustment for missing data was not required to maintain statistical integrity of the analyses, and annualized rates (primary endpoints) were calculated for each subject using all the available data. Other parameters, which provide additional data for evaluation of the patient status prior to and following conversion to GA, were reported in a nonobligatory manner. Tests of significance (signal rank test and binominal test) were used to measure changes in efficacy parameters from baseline to final examination. ARR and ARD pre- and post-conversion were analyzed using repeated measures analysis of covariance using the maximum likelihood ratio. Log transformation was implemented to the ARR and ARD to establish if there was a significant deviation from normality (i.e., $P<.001$ using the Shapiro-Wilk test). The Wilcoxon signed rank test was used within groups for EDSS, MFIS, FAMS, PASAT, and CES-D.

\section{Results}

\section{Patient disposition}

Overall, 672 patients from 148 centers across 19 countries were enrolled, with 668 patients included in the analysis (excluding four patients from Taiwan): $263 \mathrm{LA}$, 248 CWE, and 157 EE patients (Table 1). Patient characteristics were comparable at baseline between regions, 
Table 1 Baseline patient demographics, disease characteristics, and DMT history

\begin{tabular}{|c|c|c|c|}
\hline Characteristics & LA $(n=263)$ & CWE $(n=248)$ & $\mathrm{EE}(n=157)$ \\
\hline Female gender, $n(\%)$ & $189(71.9)$ & $175(70.6)$ & $108(68.8)$ \\
\hline Mean age, years ( \pm SD) & $40.1(10.1)$ & $43.0(10.2)$ & $34.7(8.4)$ \\
\hline Patients with comorbidities at recruitment, $n(\%)$ & $27(10.3)$ & $19(7.7)$ & $8(5.1)$ \\
\hline Depression & $13(4.9)$ & $6(2.4)$ & $1(0.6)$ \\
\hline Anxiety & $2(0.8)$ & $1(0.4)$ & $1(0.6)$ \\
\hline Hypertension & $1(0.4)$ & $4(1.6)$ & $2(1.3)$ \\
\hline Patients with concomitant therapies at time of recruitment, $n(\%)$ & $24(9.1)$ & $15(6.1)$ & $7(4.5)$ \\
\hline Psychoanaleptics & $14(5.3)$ & $5(2.0)$ & $1(0.6)$ \\
\hline Antiepileptics & $6(2.3)$ & $2(0.8)$ & $1(0.6)$ \\
\hline Thyroid therapy & $3(1.1)$ & $3(1.2)$ & N/A \\
\hline Mean disease duration since onset, months $( \pm S D)^{a}$ & $98.0(82.9)$ & $100.1(84.4)$ & $92.3(63.9)$ \\
\hline Mean disease duration since diagnosis, months $( \pm S D)^{b}$ & $68.9(59.6)$ & $72.1(70.7)$ & $67.9(48.5)$ \\
\hline Mean ARR, events/year $( \pm S D)^{c}$ & $1.0(0.8)$ & $0.8(0.6)$ & $0.7(0.5)$ \\
\hline \multicolumn{4}{|l|}{ Patients in ARR range, $n(\% *)$} \\
\hline $0.00-1.25$ & $166(67.5)$ & $193(84.6)$ & $130(88.4)$ \\
\hline $1.25-3.25$ & $78(31.7)$ & $35(15.4)$ & $17(11.6)$ \\
\hline$>3.25$ & $2(0.8)$ & $0(0.0)$ & $0(0.0)$ \\
\hline Data unavailable & 17 & 20 & 10 \\
\hline \multicolumn{4}{|l|}{ Clinical type of $M S, n\left(\%^{d}\right)$} \\
\hline RRMS with incomplete remissions & $171(67.6)$ & $117(47.6)$ & $91(59.1)$ \\
\hline RRMS with complete remission & $80(31.6)$ & $122(49.6)$ & $62(40.3)$ \\
\hline Clinically isolated syndrome & $0(0.0)$ & $0(0.0)$ & $1(0.6)$ \\
\hline Other & $2(0.8)$ & $7(2.8)$ & $0(0.0)$ \\
\hline Data unavailable & 10 & 2 & 3 \\
\hline \multicolumn{4}{|l|}{ Diagnosed with MS by criteria, $n\left(\%^{\mathrm{d}}\right)$} \\
\hline McDonald & $217(83.8)$ & $194(78.5)$ & $143(92.3)$ \\
\hline Poser & $42(16.2)$ & $53(21.5)$ & $12(7.7)$ \\
\hline Data unavailable & 4 & 1 & 2 \\
\hline \multicolumn{4}{|l|}{ Mobility, $n\left(\%^{d}\right)$} \\
\hline Asymptomatic & $45(19.8)$ & $46(20.0)$ & $20(14.9)$ \\
\hline Able to walk unaided for $>500 \mathrm{~m}$ & $96(42.3)$ & $126(54.8)$ & $112(83.6)$ \\
\hline Able to walk unaided for $<500 \mathrm{~m}$ & $30(13.2)$ & $27(11.7)$ & $2(1.5)$ \\
\hline Walking with bilateral support & $13(5.7)$ & $9(3.9)$ & $0(0.0)$ \\
\hline Walking with unilateral support & $33(14.5)$ & $18(7.8)$ & $0(0.0)$ \\
\hline Need of a wheelchair outdoors & $10(4.4)$ & $4(1.7)$ & $0(0.0)$ \\
\hline Data unavailable & 36 & 18 & 23 \\
\hline Mean EDSS score $( \pm S D)^{e}$ & $3.5(2.2)$ & $2.8(1.9)$ & $2.6(1.0)$ \\
\hline Mean CES-D score $(0-60)( \pm S D)^{f}$ & $16.0(11.7)$ & $16.0(10.3)$ & $20.6(19.5)$ \\
\hline Mean MFIS score $(0-84)( \pm \text { SD })^{9}$ & $32.3(19.7)$ & $31.4(19.1)$ & $33.7(27.9)$ \\
\hline Mean FAMS score $(0-176)( \pm S D)^{h}$ & $109.4(37.8)$ & $100.8(34.3)$ & $77.7(59.4)$ \\
\hline Mean PASAT score $(0-60)( \pm S D)^{i}$ & 35.6 (13.6) & 36.7 (15.7) & $51.8(5.9)$ \\
\hline
\end{tabular}


Table 1 Baseline patient demographics, disease characteristics, and DMT history (Continued)

\begin{tabular}{|c|c|c|c|}
\hline Mean observation duration, months $( \pm \mathrm{SD})^{j}$ & $20.5(6.3)$ & $18.6(7.7)$ & $19.2(7.8)$ \\
\hline \multicolumn{4}{|l|}{ Number of DMT classes used (\%) (converters only) } \\
\hline 1 & $206(85.5)$ & $201(83.4)$ & $143(92.9)$ \\
\hline 2 & $32(13.3)$ & $38(15.8)$ & $10(6.5)$ \\
\hline 3 & $3(1.2)$ & $2(0.8)$ & $1(0.6)$ \\
\hline Non-converters & 22 & 7 & 3 \\
\hline \multicolumn{4}{|l|}{ Previous type and mode of IFN- $\beta$ used, $\%^{k}$} \\
\hline IFN- $\beta-1 a$ (i.m.) & 30.3 & 36.0 & 47.4 \\
\hline IFN- $\beta-1 b$ (s.c.) & 30.3 & 25.9 & 30.5 \\
\hline IFN- $\beta-1 a$ (s.c.) & 35.5 & 30.7 & 21.4 \\
\hline \multicolumn{4}{|l|}{ Reason for conversion to $\mathrm{GA}, n(\%)^{\prime}$} \\
\hline Lack of previous DMT efficacy & $171(71.0)$ & $78(32.4)$ & $92(59.7)$ \\
\hline Presence of neutralizing antibodies & $1(0.4)$ & $44(18.3)$ & $2(1.3)$ \\
\hline Intolerable adverse events associated with previous DMT & $98(40.7)$ & $132(54.8)$ & $55(35.7)$ \\
\hline Flu-like symptoms & $67(27.8)$ & $73(30.3)$ & $40(26.0)$ \\
\hline Subjective & $29(12.0)$ & $37(15.4)$ & $17(11.0)$ \\
\hline Skin reactions & $15(6.2)$ & $14(5.8)$ & $20(13.0)$ \\
\hline Blood work & $7(2.9)$ & $18(7.5)$ & $4(2.6)$ \\
\hline Others & $21(8.7)$ & $39(16.2)$ & $4(2.6)$ \\
\hline Not specified & $1(0.4)$ & $1(0.4)$ & $0(0.0)$ \\
\hline Other & $8(3.3)$ & $21(8.7)$ & $36(23.4)$ \\
\hline Non-converters & 22 & 7 & 3 \\
\hline Discontinuation of GA, $n(\%)$ & $66(25.1)$ & $77(31.1)$ & $30(19.1)$ \\
\hline Perceived lack of efficacy by physician & $19(7.2)$ & $7(2.8)$ & $16(10.2)$ \\
\hline Perceived lack of efficacy by patient & $7(2.7)$ & $14(5.7)$ & $6(3.8)$ \\
\hline Adverse events & $9(3.4)$ & $17(6.9)$ & $4(2.5)$ \\
\hline Lost to follow-up & $14(5.3)$ & $24(9.7)$ & $2(1.3)$ \\
\hline Other & $15(5.7)$ & $8(3.2)$ & $3(1.9)$ \\
\hline
\end{tabular}

Missing data in $24 \mathrm{LA}, 28 \mathrm{CWE}$, and 5 EE patients

${ }^{\mathrm{b}}$ Missing data in $15 \mathrm{LA}, 21 \mathrm{CWE}$, and $4 \mathrm{EE}$ patients

cMissing data in $7 \mathrm{LA}, 2 \mathrm{CWE}$, and $3 \mathrm{EE}$ patients

${ }^{\mathrm{d} A d j u s t e d}$ percentage of patients with data available

e Missing data in $55 \mathrm{LA}, 9 \mathrm{CWE}$, and $8 \mathrm{EE}$ patients

${ }^{\mathrm{f}}$ Missing data in $116 \mathrm{LA}, 103 \mathrm{CWE}$, and $150 \mathrm{EE}$ patients

${ }^{9}$ Missing data in $113 \mathrm{LA}, 118 \mathrm{CWE}$, and $150 \mathrm{EE}$ patients

${ }^{\mathrm{h}}$ Missing data in $55 \mathrm{LA}, 9 \mathrm{CWE}$, and $8 \mathrm{EE}$ patients

'Missing data in 197 LA, 103 CWE, and 150 EE patients

${ }^{\mathrm{j}}$ Missing data in $11 \mathrm{LA}, 17 \mathrm{CWE}$, and $10 \mathrm{EE}$ patients

${ }^{k}$ Missing data in $32 \mathrm{LA}, 20 \mathrm{CWE}$, and $3 \mathrm{EE}$ patients

'Adjusted percentage of patients with data available. Patients were allowed to cite $\geq 1$ reason for conversion to GA. Therefore, the percentage may exceed $100 \%$

excluding EE patients, who were younger and reported fewer comorbidities and concomitant medications than LA and CWE patients. Baseline disease characteristics that were similar across regions (Table 1) included disease duration from onset, diagnosis, and mean ARR. Distribution of baseline ARR score varied slightly, with the majority of patients experiencing between 0 and 1.25 events/year. LA patients reported the highest disability (baseline EDSS) and the highest frequency of RRMS with incomplete remissions. At baseline, the majority of patients had received one previous DMT regimen in one class of agents (Table 1). Reports of flu-like symptoms were the most common reason for converting to GA. The majority of patients in all regions were converted from IFN- $\beta$ therapy.

\section{Annualized relapse rate}

ARR was significantly decreased in all groups following the conversion to GA (Fig. 1). LA patients, who had the highest baseline ARR rate, also had the greatest ARR 


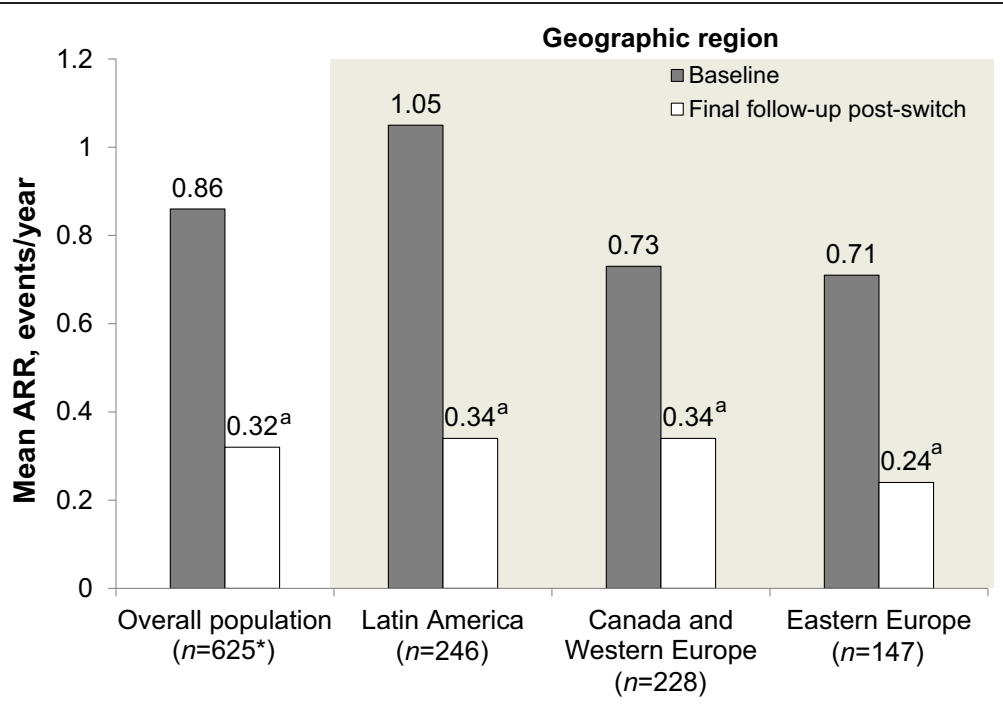

Fig. 1 Reduction of annualized relapse rate by geographic region. ${ }^{a} P<.0001$ vs. baseline. ${ }^{*}$ The overall population size does not each the sum of the three individual regions due to four patients in Taiwan that excluded from the regional analysis. ARR annualized relapse rate

reduction $(1.05 \pm 0.78$ pre-conversion to $0.34 \pm 0.86$ post-conversion; $P<.0001$, Shapiro-Wilk test). ARR went from $0.73 \pm 0.58$ to $0.34 \pm 0.84(P<.0001)$ in CWE patients and $0.71 \pm 0.50$ to $0.24 \pm 0.92(P<.0001)$ in EE patients.

\section{Disease progression}

In total, 499 patients had $\geq 1$ EDSS assessment after baseline examination, with the overall population showing a significant increase in EDSS score (i.e., progression to worse disability) from 2.9 at baseline to 3.02 at final follow-up, post-switch $(P=.0256)$. There was a significant difference between regions in the degree of change in EDSS score while on GA therapy $(P=.0230$, Wilcoxon signed-rank test), driven by a significant increase in CWE patients' EDSS score of $0.26 \pm 1.18(P=.0016)$. Neither LA nor EE patients had significant changes in mean EDSS score from baseline. Improved (i.e., numerically lowered) EDSS scores were seen in $32.5 \%, 24.4 \%$, and $33.8 \%$ of LA, CWE, and EE patients, respectively. There was no change from baseline in $35.8 \%, 34.9 \%$, and $41.2 \%$, respectively. Deterioration was reported in $31.8 \%$, $40.7 \%$, and $25.0 \%$, respectively.

\section{Disease activity}

Disease activity while receiving GA varied. LA patients reported the highest incidence of frequent exacerbations and of fast progression of MS (Table 2). Fewer LA patients reported rarely experiencing exacerbations (27.9\%, vs. $43.5 \%$ CWE and $66.0 \%$ EE patients).

\section{Change in mobility}

The majority of patients showed no change in mobility scores $(63.4 \%, 62.8 \%$, and $67.5 \%$ of LA, CWE, and EE patients, respectively). Mobility scores improved in $17.1 \%$ LA and $18.9 \%$ CWE patients, with a significant improvement in $23.0 \%$ of EE patients $(P=.0079)$. Mobility scores deteriorated in $19.4 \%, 18.4 \%$, and $9.5 \%$ of LA, CWE, and EE patients, respectively. Data were missing for $47 \mathrm{LA}, 52 \mathrm{CWE}$, and $31 \mathrm{EE}$ patients.

Table 2 MS disease activity over the 2-year study period

\begin{tabular}{llll}
\hline Patients, $n(\%)$ & LA & CWE & $\begin{array}{l}\text { EE } \\
(n=153)\end{array}$ \\
\hline Stable MS (Stage 1) & $(n=251)$ & $47(19.1)$ & $22(14.4)$ \\
Rare exacerbations ( $\leq 1$ per year, Stage 2a) & $37(14.7)$ & $107(43.5)$ & $101(66.0)$ \\
Slow progression ( $\leq 0.5$ EDSS points per year, Stage 2b) & $70(27.9)$ & $35(14.2)$ & $6(3.9)$ \\
Frequent exacerbations (>1 per year, Stage 3a) & $48(19.1)$ & $43(17.5)$ & $21(13.7)$ \\
Fast progression (>0.5 EDSS points per year, Stage 3b) & $80(31.9)$ & $3(1.2)$ & $1(0.7)$ \\
Not classified/not available & $11(4.4)$ & $11(4.5)$ & $2(1.3)$ \\
\hline
\end{tabular}




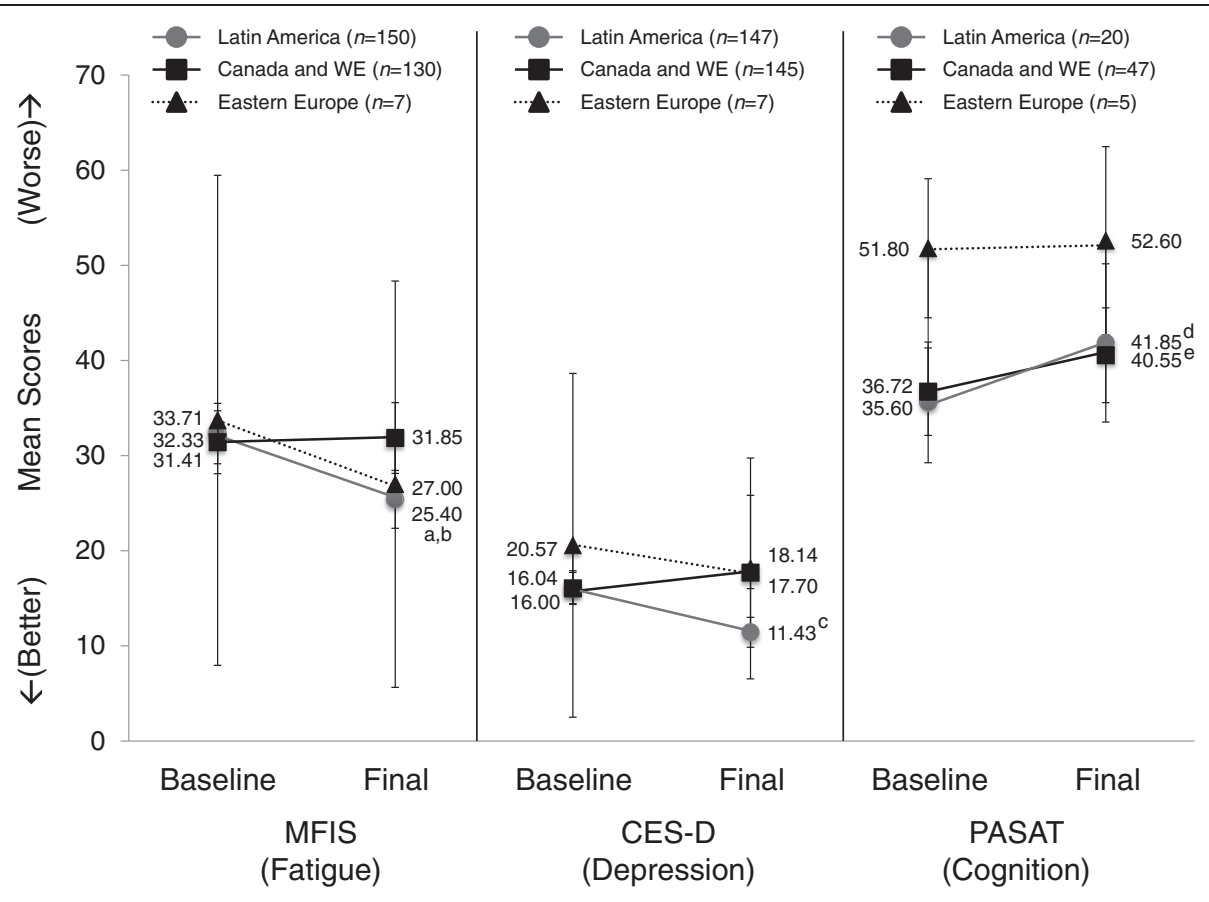

Fig. 2 Change in fatigue, depression, and cognition scores by geographic region. ${ }^{a} P<.0001$ for Latin America vs. Canada/Western Europe at final follow-up. ${ }^{b} P<.0001$ for change from baseline. ${ }^{c} P<.0001$ for change from baseline. ${ }^{d} P=.0030$ for change from baseline. ${ }^{e} P=.0024$ for change from baseline. CES-D Centre for Epidemiologic Studies Depression, MFIS Modified Fatigue Impact Scale, PASAT Paced Auditory Serial Addition Test, WE Western Europe

\section{Secondary endpoints}

In LA patients, there was a significant improvement in the mean difference in MFIS score from baseline $(6.9 \pm 15.4$; $P<.0001)$ (Fig. 2). EE patients and CWE patients experienced a nonsignificant increase from baseline of $6.7 \pm 18.2$ and $0.4 \pm 13.6$, respectively.

Only LA patients showed a significant change in QoL, with an improvement in mean FAMS score of $18.5 \pm$ 46.5 from baseline $(P=.0008)$. CWE patients had a mean FAMS increase of $0.6 \pm 20.5$, while EE patients showed a decrease of $2.3 \pm 8.8$ (Fig. 3).

LA patients reported a significant improvement in depression symptoms, with a decrease in mean CES-D score of $4.6 \pm 10.9(P<.0001)$ (Fig. 2). CWE patients showed a numerical increase in mean CES-D score of $1.7 \pm 9.9$, while EE patients showed a decrease of $2.4 \pm$ 10.8. These changes were not statistically significant.

Patients from all regions showed an improvement in mean PASAT (cognition) scores (Fig. 2). LA patients had the greatest improvement, with a statistically significant mean increase of $6.3 \pm 9.1$ in PASAT score $(P=.0030)$. CWE patients also showed a statistically significant mean increase of $3.8 \pm 9.6(P=.0024)$, while EE patients had a smaller mean increase of $0.8 \pm 5.3$ (NS).

LA patients reported the highest efficacy with GA than the previous drug $(70.0 \% ; P<.0001)$, followed by EE and CWE patients (42.4\% and $41.6 \%$, respectively; both
$P<.0001)$. LA patients reported the lowest percentage of "feeling worse" on GA therapy (3.9\%), with EE patients reporting the highest percentage $(15.2 \%)$, followed by CWE patients $(6.1 \%)$. There was no change in perception of efficacy in $26.2 \%, 52.2 \%$ and $42.4 \%$ of LA, CWE, and EE patients, respectively (Additional file 1).

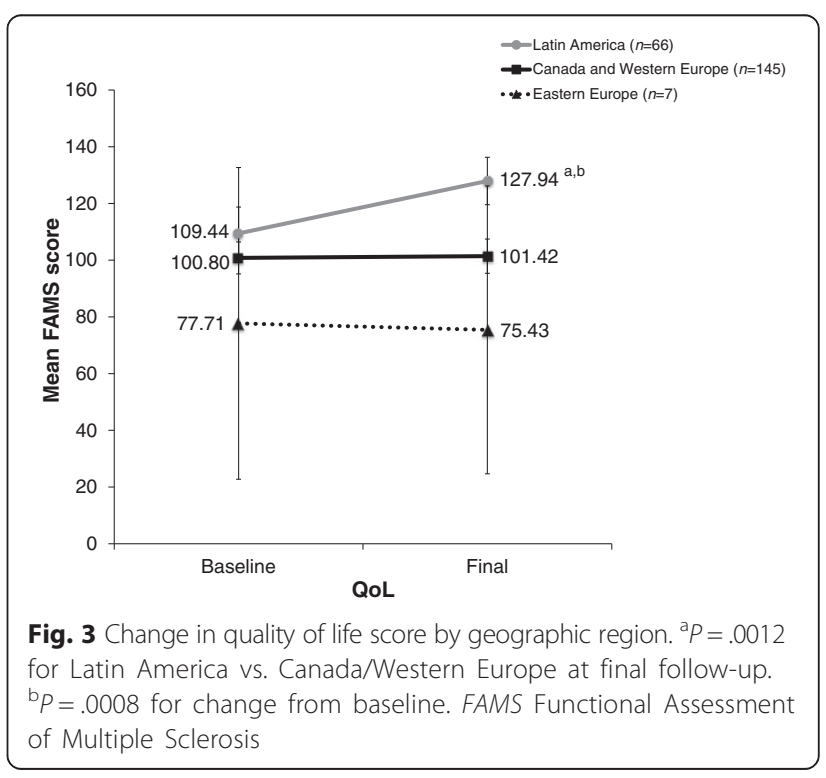




\section{Safety}

AEs reported in all three regions are listed in Table 3. Patients from all regions experienced statistically significant improvements in AEs with GA use $(P<.0001)$.

\section{Discussion}

Converting to another class of immunomodulatory therapy represents one treatment strategy in MS patients who fail to respond adequately to first-line treatments [10]. However, this strategy may not always be beneficial because of geographical variations in treatment regimens and therapeutic strategies. For example, GA and IFN- $\beta$ are typically the first-line treatment options in MS treatment algorithms, regardless of the geographical region [11]. However, some LA neurologists prescribe azathioprine because of limited DMT access or because the drugs are unavailable on healthcare plans [16, 17]. Expert guidance on the specific steps of a conversion have begun to be published, suggesting a conversion in therapy may be considered when there is a high level of concern about relapse rates, progression of MS and magnetic resonance imaging outcomes, a medium level of concern about any two factors, or a low level of concern about all three factors [10]. The general nature of the guidelines is due to inconsistent results with converting.

In this sub-analysis of the COPTIMIZE trial, converting to GA was well tolerated, reduced disease progression and activity, and improved other secondary endpoints in patients across all three regions to varying degrees. LA patients experienced the largest reduction in ARR; however, their baseline ARR was much higher than CWE and EE patients. Ultimately, all three regions reached similar ARR. LA patients had higher baseline rates of comorbidities and incomplete previous remissions than the other two regions, as well as significant improvements in QoL, depression, fatigue, and cognition scores. They also had a better perception of the benefits of a GA conversion in terms of efficacy and AEs than CWE and EE patients.

These discrepancies may be due to differences in healthcare standards and environmental factors between

Table 3 Adverse events (AEs)

\begin{tabular}{|c|c|c|c|}
\hline Patients, events/patients $\left(\%{ }^{\mathrm{a}}\right)$ & LA $(n=263)$ & CWE $(n=248)$ & $\mathrm{EE}(n=157)$ \\
\hline \multicolumn{4}{|l|}{ Most common AEs by preferred term } \\
\hline Dyspnea & $5 / 4(1.5)$ & $4 / 3(1.2)$ & $1 / 1(0.6)$ \\
\hline Syncope & $2 / 2(0.8)$ & $2 / 2(0.8)$ & $0 / 0(0.0)$ \\
\hline Injection site reaction & $2 / 2(0.8)$ & $8 / 8(3.2)$ & $0 / 0(0.0)$ \\
\hline Injection site pain & $2 / 2(0.8)$ & $14 / 10(4.0)$ & 0/0 (0.0) \\
\hline Injection site induration & $1 / 1(0.4)$ & $5 / 5(2.0)$ & 0/0 (0.0) \\
\hline Fatigue & $1 / 1(0.4)$ & $3 / 3(1.2)$ & 0/0 (0.0) \\
\hline Arthralgia & 1/1 (0.4) & $3 / 3(1.2)$ & $0 / 0(0.0)$ \\
\hline Rash & 0/0 (0.0) & $2 / 2(0.8)$ & $2 / 2(1.3)$ \\
\hline Anxiety & 0/0 (0.0) & $1 / 1(0.4)$ & $1 / 1(0.6)$ \\
\hline \multicolumn{4}{|l|}{ Most common AE classified by system organ class } \\
\hline General disorders and administration site conditions & $16 / 13(4.9)$ & $55 / 32(12.9)$ & $5 / 4(2.6)$ \\
\hline Nervous system disorders & $10 / 10(3.8)$ & $9 / 8(3.2)$ & $0 / 0(0.0)$ \\
\hline Respiratory, thoracic, and mediastinal disorders & $7 / 6(2.3)$ & $4 / 3(1.2)$ & $0 / 0(0.0)$ \\
\hline \multicolumn{4}{|l|}{ Severity of $A E$, events/patients } \\
\hline Mild & 19/12 (4.6) & $40 / 28(11.3)$ & $0 / 0(0.0)$ \\
\hline Moderate & $22 / 12(4.6)$ & $62 / 38(15.3)$ & $5 / 5(3.2)$ \\
\hline Severe & $13 / 6(2.3)$ & 14/9 (3.6) & $5 / 3(1.9)$ \\
\hline Data unavailable & $4 / 2(0.8)$ & $7 / 5(2.0)$ & $2 / 2(1.3)$ \\
\hline \multicolumn{4}{|c|}{ Patient-reported assessment of AEs after glatiramer acetate treatment ${ }^{b}$} \\
\hline Improved with glatiramer acetate & $209(80.4)$ & $162(66.1)$ & $58(38.4)$ \\
\hline Same with glatiramer acetate & $41(15.8)$ & $62(25.3)$ & $87(57.6)$ \\
\hline Worse with glatiramer acetate & $10(3.9)$ & $21(8.6)$ & $6(4.0)$ \\
\hline Data unavailable & 3 & 3 & 6 \\
\hline
\end{tabular}

a Percentage reported as the proportion of patients experiencing events

${ }^{\mathrm{b}}$ Adjusted percentage of patients with data available 
the different regions. For example, the US, Canada, and LA have clinical treatment guidelines that vary in the topics discussed and the use of GA, IFN- $\beta$, natalizumab, dalfampridine, and fingolimod [10, 16-18]. Also, it is possible that patients from different regions may have different epidemiological characteristics and comorbidities [16]. These regional differences cannot be adjusted in such a study, where the observational, non-interventional design carries inherent analytical limitations.

Now that consensus guidelines have defined a suboptimal treatment response, and neurodegenerative activity has been identified even in early stages of disease [19, 20], converting to another DMT class represents a logical treatment strategy in patients who fail to respond adequately to first-line treatments. Our results suggest that more attention is required regarding the importance of establishing formal conversion algorithms that account for geographic variability, ensuring that all patients who could benefit from such an approach are managed in a timely and optimal manner.

Despite study limitations, our observations emphasize the importance of changing a therapy regimen, in particular IFN-based, to improve efficacy and/or overcome treatment intolerance that would otherwise compromise compliance. This is in alignment with previous studies of this strategy $[9,21]$.

\section{Conclusions}

All patients experienced significant improvements in ARR regardless of geographic region. There were differences between regions in patients' baseline parameters, comorbidities, and reasons for converting to GA treatment. The evolution of guidelines regarding suboptimal treatment response and DMT conversion has the potential to affect strategies for monitoring and treating patients across all geographies and to improve clinical and patient-reported outcomes.

\section{Additional files}

Additional file 1: Patient perception of glatiramer acetate treatment efficacy by geographic region. ${ }^{a} P<.0001$ for Latin America vs. other geographic areas. (PDF 7 kb)

Additional file 2: COPITIMIZE: Main Investigator sites. (DOCX 14 kb)

\footnotetext{
Abbreviations

AE: Adverse event; ARD: Annualized rate of deterioration; ARR: Annualized relapse rate; CES-D: Centre for Epidemiologic Studies Depression; CWE: Canada and Western Europe; DMT: Disease-modifying therapy; EDSS: Expanded Disability Status Scale; EE: Eastern Europe; FAMS: Functional Assessment of Multiple Sclerosis; GA: Glatiramer acetate; IFN: Interferon; i.m.: Intramuscular; LA: Latin America; MFIS: Modified Fatigue Impact Scale; MS: Multiple sclerosis; PASAT: Paced Auditory Serial Addition Test; QoL: Quality of life; RMS: Relapsing-remitting multiple sclerosis; S.c.: Subcutaneous; SD: Standard deviation.
}

\section{Competing interests}

TZ has received speaker honoraria from Almirall, Bayer-Schering, Biogen Idec, Genzyme, GSK, Sanofi-Aventis, Merck Serono, MSD, Novartis, and Teva. He serves as a consultant for Bayer-Schering, Biogen Idec, Novartis, and Teva, and he receives research support from the Deutsche Diabetes Stiftung, Hertie Foundation, the Robert Pfleger Foundation, and the Roland Ernst Foundation. YG-S is an employee of Teva Pharmaceutical Industries.

\section{Authors' contributions}

$\mathrm{TZ}$ and YG-S contributed equally to the development of this manuscript and have approved the manuscript for submission.

Authors' information

Not applicable.

\section{Acknowledgements}

The Authors would like to thank Rhonda Charles, PhD and Bryan Sepulveda, $\mathrm{PhD}$, who provided editorial support with funding from Teva

Pharmaceuticals Industries Ltd., Petach Tikva, Israel. The participating centers are listed in Additional file 2.

\section{Role of the funding source}

This study was funded locally by Teva affiliates for database administration. Teva Pharmaceuticals Industries Ltd. funded the study design, collection, interpretation, and analysis of data, and development and submission of this manuscript.

\section{Author details}

${ }^{1}$ Center of Clinical Neuroscience, Neurological University Clinic, University Clinic Carl Gustav Carus, University of Technology Dresden, Fetscherstraße 74, D-01307 Dresden, Germany. ${ }^{2}$ Teva Pharmaceutical Industries Ltd, 5 Basel Street, Petah Tikva 49131, Israel.

Received: 20 March 2015 Accepted: 29 September 2015

Published online: 08 October 2015

\section{References}

1. Aharoni R. The mechanism of action of glatiramer acetate in multiple sclerosis and beyond. Autoimmun Rev. 2013;12:543-53.

2. Koch-Henriksen N, Sørensen PS. The changing demographic pattern of multiple sclerosis epidemiology. Lancet Neurol. 2010;9:520-32.

3. Polman CH, O'Connor PW, Havrdova E, Hutchinson M, Kappos L, Miller DH, et al. A randomized, placebo-controlled trial of natalizumab for relapsing multiple sclerosis. N Engl J Med. 2006;354:899-910.

4. Comi G, Filippi M, Wolinsky JS. European/Canadian multicenter, double-blind, randomized, placebo-controlled study of the effects of glatiramer acetate on magnetic resonance imaging-measured disease activity and burden in patients with relapsing multiple sclerosis. European/Canadian Glatiramer Acetate Study Group. Ann Neurol. 2001;49:290-7.

5. The IFNB, Group MSS. Interferon beta- $1 b$ is effective in relapsing-remitting multiple sclerosis. I. Clinical results of a multicenter, randomized, doubleblind, placebo-controlled trial. Neurology. 1993;43:655-61.

6. Rudick RA, Stuart WH, Calabresi PA, Confavreux C, Galetta SL, Radue EW, et al. Natalizumab plus interferon beta-1a for relapsing multiple sclerosis. N Engl J Med. 2006;354:911-23.

7. Coyle PK. Switching algorithms: from one immunomodulatory agent to another. J Neurol. 2008;255 Suppl 1:44-50.

8. Goodin DS, Frohman EM, Hurwitz B, O'Connor PW, Oger JJ, Reder AT, et al. Neutralizing antibodies to interferon beta: assessment of their clinical and radiographic impact: an evidence report: report of the Therapeutics and Technology Assessment Subcommittee of the American Academy of Neurology. Neurology. 2007;68:977-84.

9. Rio J, Tintore M, Sastre-Garriga J, Nos C, Castilló J, Tur C, et al. Change in the clinical activity of multiple sclerosis after treatment switch for suboptimal response. Eur J Neurol. 2012;19:899-904.

10. Freedman MS, Selchen D, Arnold DL, Prat A, Banwell B, Yeung M, et al Treatment optimization in MS: Canadian MS Working Group updated recommendations. Can J Neurol Sci. 2013;40:307-23.

11. Hartung HP, Montalban X, Sorensen PS, Vermersch P, Olsson T. Principles of a new treatment algorithm in multiple sclerosis. Expert Rev Neurother. 2011;11:351-62. 
12. Johnson KP. Glatiramer acetate for treatment of relapsing-remitting multiple sclerosis. Expert Rev Neurother. 2012;12:371-84.

13. Scott LJ. Glatiramer acetate: a review of its use in patients with relapsingremitting multiple sclerosis and in delaying the onset of clinically definite multiple sclerosis. CNS Drugs. 2013;27:971-88.

14. Ziemssen T, Bajenaru OA, Carrá A, de Klippel N, de Sá JC, Edland A, et al. A 2-year observational study of patients with relapsing-remitting multiple sclerosis converting to glatiramer acetate from other disease-modifying therapies: the COPTIMIZE trial. J Neurol. 2014;261:2101-11.

15. Kulesher R, Forrestal E. International models of health systems financing. J Hosp Admin. 2014;3:127-39.

16. Carrá A, Macias-slas MA, Gabbai AA, Correale J, Bolaña C, Sotelo ED, et al. Optimizing outcomes in multiple sclerosis: consensus guidelines for the diagnosis and treatment of multiple sclerosis in Latin America. Ther Adv Neurol Disord. 2011;4:349-60.

17. Finkelsztejn A, Fragoso YD, Ferreira ML, Lana-Peixoto MA, Alves-Leon SV, Gomes S, et al. The Brazilian database on pregnancy in multiple sclerosis. Clin Neurol Neurosurg. 2011;113:277-80.

18. Miller RM, Happe LE, Meyer KL, Spear RJ. Approaches to the management of agents used for the treatment of multiple sclerosis: consensus statements from a panel of U.S. managed care pharmacists and physicians. J Manag Care Pharm. 2012;18:54-62.

19. Filippi M, Bozzali M, Rovaris M, Gonen O, Kesavadas C, Ghezzi A, et al. Evidence for widespread axonal damage at the earliest clinical stage of multiple sclerosis. Brain. 2003;126(Pt 2):433-7.

20. De Stefano N, Narayanan S, Francis GS, Arnaoutelis R, Tartaglia MC, Antel JP, et al. Evidence of axonal damage in the early stages of multiple sclerosis and its relevance to disability. Arch Neurol. 2001;58:65-70.

21. Gajofatto A, Bacchetti P, Grimes B, High A, Waubant E. Switching first-line disease-modifying therapy after failure: impact on the course of relapsingremitting multiple sclerosis. Mult Scler. 2009;15:50-8.

\section{Submit your next manuscript to BioMed Central and take full advantage of:}

- Convenient online submission

- Thorough peer review

- No space constraints or color figure charges

- Immediate publication on acceptance

- Inclusion in PubMed, CAS, Scopus and Google Scholar

- Research which is freely available for redistribution 